\title{
Planejamento e apoio no processo de trabalho das equipes de atenção básica no Nordeste: análise do PMAQ-AB ( $3^{\circ}$ ciclo)
}

\author{
Planning and support in the work process of PHC teams \\ in the Northeast: analysis of the PMAQ-AB (3rd cycle)
}

\author{
Gladston Thalles da Silva (https://orcid.org/0000-0002-1315-2770) ${ }^{1}$ \\ Ferdinando Oliveira Carvalho (https://orcid.org/0000-0003-0306-5910) ${ }^{1}$ \\ Anya Pimentel Gomes Fernandes Vieira-Meyer (https://orcid.org/0000-0003-4237-8995) ${ }^{2}$ \\ Gessyka Mayara Soares Gomes (https://orcid.org/0000-0003-4651-937X) ${ }^{1}$ \\ Lis Maria Machado Ribeiro Bezerra (https://orcid.org/0000-0002-1192-057X) ${ }^{1}$ \\ Rayssa Veras Camelo (https://orcid.org/0000-0002-6822-1163) ${ }^{3}$ \\ Valter Cordeiro Barbosa Filho (https://orcid.org/0000-0002-4769-4068) ${ }^{4}$
}

\footnotetext{
${ }^{1}$ Universidade Federal do Vale do São Francisco. Av. José de Sá Maniçoba s/n, Centro. 56304-205 Petrolina PE Brasil. gladstonthalles@bol.com.br ${ }^{2}$ Escritório Fiocruz Ceará, Fundação Oswaldo Cruz. Fortaleza CE Brasil. ${ }^{3}$ Universidade Estadual do Ceará. Fortaleza CE Brasil. ${ }^{4}$ Instituto Federal do Ceará. Aracati CE Brasil.
}

\begin{abstract}
This paper aims to describe the indicators related to planning and support in the work process reported by the Family Health Teams, from the 3rd cycle of the National Program for Improving Access and Quality of Primary Care (PMAQ-AB) in the Brazilian Northeast. This cross-sectional study employed secondary data from the external evaluation of the $3 r d$ cycle of the PMAQ-AB. Sixteen indicators were used to ascertain the teams' work process actions, and 14,489 family health teams that adhered to the program were evaluated. Among the indicators assessed, we found that the teams held a meeting and planned actions and self-evaluated, monitored, and analyzed health indicators to reorganize the work process, highlighting the determining and conditioning factors. Health surveillance and the Extended Family Health Center (NASF) stand out in the indicators of institutional support and multidisciplinary support for solving complex cases. We identified improvements in the work process of the teams in the Northeast region, but the reality of fragmented and vertical work still requires a change to promote an outlook of shared teamwork, directly influencing the work process. Key words Primary Health Care, Family Health Strategy, Health Assessment
\end{abstract}

Resumo O objetivo deste artigo é descrever os indicadores referentes ao planejamento e apoio no processo de trabalho reportados pelas Equipes de Saúde da Família, a partir do $3^{\circ}$ ciclo do Programa Nacional de Melhoria do Acesso e da Qualidade da Atenção Básica (PMAQ-AB) na região Nordeste do Brasil. Estudo transversal, utilizando dados secundários da avaliação externa do $3^{\circ}$ ciclo do PMAQ-AB. Dezesseis indicadores foram utilizados para averiguar as ações do processo de trabalho das equipes. Avaliaram-se 14.489 equipes de saúde da família que aderiram ao programa. Dentre os indicadores avaliados, verificou-se que as equipes realizaram reunião e planejamento das ações, autoavaliação, monitoramento e análise dos indicadores de saúde para reorganização do processo de trabalho, evidenciando os fatores determinantes e condicionantes. Nos indicadores de apoio institucional e apoio matricial para resolução de casos complexos, destacam-se a vigilância em saúde e o Núcleo Ampliado de Saúde da Família (NASF). Identificou-se que ocorreram melhorias no processo de trabalho das equipes na região Nordeste, porém, ainda precisa mudar a realidade de trabalho fragmentada e verticalizada, favorecendo um panorama de trabalho em equipe e compartilhado, influenciando diretamente no processo de trabalho.

Palavras-chave Atenção Primária à Saúde, Estratégia Saúde da Família, Avaliação em Saúde 


\section{Introdução}

A Atenção Primária à Saúde (APS) é a alternativa para resolver de forma sustentável os desafios atuais do sistema de saúde, sendo fundamental para alcançar as metas globais compartilhadas na cobertura universal de saúde, bem como os Objetivos Para o Desenvolvimento Sustentável (ODS) relacionados à saúde por sua capacidade de alcance da cobertura universal, favorecendo ações intersetoriais e equidade, no intuito de assegurar uma vida saudável e promover o bem-estar em todas as idades, de acordo com a Declaração de Astana ${ }^{1}$.

Nesse contexto, a consolidação da APS nas últimas décadas representa um dos avanços mais relevantes do Sistema Único de Saúde (SUS), enquanto política pública e sistema de saúde universal no Brasil. Tal ascensão está ancorada especialmente com a implantação da estratégia saúde da família (ESF) e com a institucionalização da avaliação da atenção básica $(\mathrm{AB})$ pelo Ministério da Saúde (MS). Tais iniciativas vêm provocando aumento da oferta de ações e serviços de amplo espectro e concorrendo para efeitos positivos importantes sobre a saúde da população, marcando uma das inovações mais significativas na APS desde a Declaração de Alma-Ata e a criação do SUS².

Nesse contexto, a Política Nacional da Atenção Básica (PNAB) afirma que o planejamento é uma ferramenta de gestão indispensável utilizada pelas equipes que sustentam a organização dos diversos e complexos processos de trabalho na AB. Propondo uma ação futura, o planejamento expressa propósitos e interesses em disputa, bem como a criatividade, participação e independência, estando aceitável a mudança $a^{3,4}$.

Destaca-se ainda a importância do planejamento de ações com base no diagnóstico situacional do território de atuação da Equipe de Saúde da Família (EqSF) e a realização do trabalho com território definido para manter vínculo com a comunidade, baseado nas realidades existenciais, com atuação conjunta das equipes multiprofissionais para melhores resultados no processo de autoavaliação $0^{5,6}$.

$\mathrm{Na}$ autoavaliação da equipe, a qualidade da $\mathrm{AB}$ pode ser mensurada pelo consenso dos profissionais da equipe, potencialmente habilitados para avaliar a realidade do processo de trabalho no contexto em que estão inseridos. Tal análise também pode facilitar o processo de decisão e organização das práticas de cuidado, contribuindo para o fortalecimento da ESF através do apoio e planejamento 6 .
No ano de 2011 foi implantado no Brasil o Programa Nacional de Melhoria do Acesso e da Qualidade da Atenção Básica (PMAQ-AB) pelo Ministério da Saúde. Este é um modelo de avaliação de desempenho dos sistemas de saúde, que pretende mensurar os possíveis efeitos da política de saúde visando subsidiar a tomada de decisão baseada no planejamento e apoio institucional das ações, garantindo a transparência dos processos de gestão do SUS e dando visibilidade aos resultados alcançados, fortalecendo o controle social e o foco nos usuários ${ }^{7}$.

O PMAQ-AB visa a ampliação do acesso da população aos serviços, a melhoria das condições de trabalho e, principalmente, da qualidade da atenção. Nesse contexto, um dos aspectos avaliados pelo programa é o processo de trabalho das equipes de $\mathrm{AB}^{8}$.

Considerando a amplitude da proposta do PMAQ- $A B$, nota-se que ainda é incipiente a inclusão de práticas avaliativas pelas equipes de atenção básica no país. Também há carência de estudos de maior abrangência capazes de revelarem a realidade do processo de trabalho, variando de acordo com as peculiaridades de cada município, estado ou região que deve ser considerado no processo avaliativo. Desse modo, torna-se pertinente a importância de considerar os dados da região Nordeste referentes ao planejamento e apoio reportados pelos profissionais de saúde da família no intuito de compreender quais ações são mais realizadas ou que necessitam de aprimoramento no processo de trabalho.

Nesse sentido, o presente trabalho tem por objetivo descrever os indicadores referentes ao planejamento e apoio no processo de trabalho reportados pelos profissionais das EqSF, a partir dos dados do $3^{\circ}$ ciclo do PMAQ-AB na região Nordeste do Brasil.

\section{Métodos}

Trata-se de um estudo transversal, em que foram utilizados dados secundários, cuja coleta foi realizada de forma multicêntrica, envolvendo as equipes de atenção básica que atuavam em municípios do Nordeste brasileiro e que aderiram ao 3o (2017) ciclo do PMAQ-AB. Para este estudo, foram consideradas 14.489 equipes de atenção básica da região Nordeste, correspondendo a $37,28 \%$ do total das equipes avaliadas no país e 99,94\% das equipes do Nordeste.

O estudo foi realizado considerando a região Nordeste do país, abrangendo todo o território 
macrorregional composto por nove estados: Alagoas, Bahia, Ceará, Maranhão, Paraíba, Pernambuco, Piauí, Rio Grande do Norte e Sergipe. Este é o terceiro maior complexo regional do país, ocupando $18,2 \%$ de sua área total ${ }^{9}$.

Os dados do presente estudo foram retirados da etapa de certificação, que contempla a fase da avaliação externa, momento em que um grupo de entrevistadores devidamente capacitados aplicaram instrumentos avaliativos para verificação de padrões de acesso e qualidade da $\mathrm{AB}$ alcançados pelos municípios, sendo conduzida por instituições de ensino e/ou pesquisa contratadas pelo MS. Portanto, o instrumento foi aplicado na própria UBS pelo entrevistador junto a um profissional da ESF designado pelos próprios membros da equipe para responder as questões. Os dados coletados foram transferidos em seguida para o banco de dados nacional do MS. Salientase que os dados referentes à variável de contexto listada acima foram retirados do website do Departamento de $\mathrm{AB}$ do MS, tendo como base os resultados da Avaliação externa do $3^{\circ}$ Ciclo do PMAQ-AB.

A avaliação externa foi desenvolvida em seis módulos distintos. Neste estudo, os dados são oriundos do módulo II - entrevista com o profissional da equipe de Atenção Básica e verificação de documentos na Unidade Básica de Saúde -, especificamente retirados das questões referentes às ações do processo de trabalho, como planejamento da equipe e apoio institucional; acesso das equipes ao NASF; articulação entre ESF e NASF; apoio matricial para as equipes de $\mathrm{AB}$. O questionário continha dezesseis perguntas relativas à realização de reunião de equipe e periodicidade; planejamento das ações; consideração de resultados alcançados nos ciclos anteriores do PMAQ-AB na organização do processo de trabalho; realização de processo autoavaliativo; informações de situações de saúde da população disponibilizadas pela gestão para auxiliar a equipe na análise de situação; planejamento e organização do cronograma de ações do NASF em conjunto com a equipe; recebimento de apoio institucional permanente de uma equipe ou pessoa da secretaria municipal de saúde, objetivando discutir o processo de trabalho ou auxiliar nos problemas detectados; avaliar o trabalho conjunto do apoiador institucional com a equipe; averiguar se a equipe recebe apoio de outros profissionais em casos complexos e quais os profissionais que realizam o apoio matricial; revelar em qual situação a equipe sente a necessidade de apoio do NASF e quais formas de solicitar; verificar se as soli- citações são atendidas em tempo adequado; investigar a existência de critérios definidos e pactuados entre equipes e o NASF para acionar em situações imprevistas e se ocorre o apoio nessas eventualidades.

A análise descritiva foi utilizada para apresentação dos dados, considerando a frequência absoluta, frequência relativa e intervalo de confiança de 95\% (IC95\%). As análises foram realizadas utilizando o programa estatístico Stata ${ }^{\circledR}$ v.13.0.

A pesquisa faz parte do projeto de pesquisa intitulado "Avaliação da Atenção Básica no Brasil: estudos multicêntricos integrados sobre acesso, qualidade e satisfação dos usuários", aprovado pelo Comitê de Ética em Pesquisa da Universidade Federal do Rio Grande do Sul.

\section{Resultados}

Atualmente, existem cadastradas 16.497 EqSF credenciadas pelo MS na região Nordeste do país. Desse quantitativo, foram certificadas no $3^{\circ}$ ciclo do PMAQ-AB 14.489 equipes, com adesão de $97,89 \%$ dos municípios do Nordeste ao programa.

Considerando a amostra total da região Nordeste, as ações das EqSF voltadas ao planejamento com maiores proporções foram: reunião de equipe $(95,2 \%)$, periodicidade de reunião mensal $(42,2 \%)$, monitoramento e análise dos indicadores e informações de saúde $(87,6 \%)$, realização de processo de autoavaliação $(87,2 \%)$ e organização do cronograma e planejamento das ações do NASF em conjunto com as equipes $(68,3 \%)$ (Tabela 1).

Constatou-se que 91,2\% (IC95\%: 90,7-91,6) das equipes reportaram receber apoio permanente da gestão municipal. Os dados mostram que 93,9\% (IC95\%: 93,5-94,3) das EqSF recebem apoio de outros profissionais para resolução de casos considerados complexos. As principais ações de apoio matricial são da: vigilância em saúde $(83,2 \%)$ e NASF $(80,3 \%)$ (Tabela 2$)$.

\section{Discussão}

Os resultados do presente estudo evidenciaram que a maior parte das equipes realiza atividades para planejamento das ações e reunião com periodicidade mensal. Pesquisa que analisou o processo de trabalho das equipes a nível nacional e estadual também constataram que a maioria 
Tabela 1. Descrição das EqSF relacionados aos indicadores de planejamento na região Nordeste, com dados do PMAQ-AB, 2017.

\begin{tabular}{lrr}
\hline \multicolumn{1}{c}{ Indicadores de Planejamento } & \multicolumn{2}{c}{ Região Nordeste } \\
\cline { 2 - 3 } & \multicolumn{1}{c}{ No } & \% (IC95\%) \\
\hline Realiza reunião de equipe & 13.799 & $95,2(94,9-95,6)$ \\
Periodicidade das reuniões & & \\
$\quad$ Semanal & 3.379 & $23,3(22,6-24,0)$ \\
$\quad$ Quinzenal & 3.797 & $26,2(25,5-26,9)$ \\
$\quad$ Mensal & 6.120 & $42,2(41,4-43,0)$ \\
$\quad$ Sem periodicidade & 503 & $3,50(3,20-3,80)$ \\
A equipe realiza atividade para planejamento das ações & 13.600 & $93,9(93,5-94,2)$ \\
A equipe realiza o monitoramento e análise dos indicadores de & 12.694 & $87,6(87,0-88,1)$ \\
informações em saúde & & \\
Resultados alcançados em ciclos anteriores do PMAQ-AB foram & 10.006 & $69,0(68,3-69,8)$ \\
considerados na organização do processo de trabalho & & \\
No último ano, a equipe realizou algum processo de autoavaliação & 12.641 & $87,2(86,7-87,7)$ \\
$\begin{array}{l}\text { A gestão disponibiliza informações que auxiliem na análise de situação de } \\
\text { saúde da população }\end{array}$ & 13.072 & $90,2(89,7-90,7)$ \\
$\begin{array}{l}\text { O planejamento e organização do cronograma de ações do NASF são } \\
\text { realizados de forma conjunta com as equipes }\end{array}$ & 9.902 & $68,3(67,9-69,0)$ \\
\hline
\end{tabular}

Fonte: Dados elaborados pelos autores a partir dos resultados finais do 3º ciclo do PMAQ/MS, 2017.

Tabela 2. Descrição da quantidade de EqSF relacionados aos indicadores de acesso, apoio institucional e apoio do NASF na região Nordeste, com dados do PMAQ-AB, 2017.

\begin{tabular}{|c|c|c|}
\hline \multirow{2}{*}{ Indicadores de Acesso, Apoio Institucional e Apoio do NASF } & \multicolumn{2}{|c|}{ Região Nordeste } \\
\hline & No & $\%(\mathrm{IC} 95 \%)$ \\
\hline $\begin{array}{l}\text { EqSF recebe apoio institucional permanente para discutir o processo de } \\
\text { trabalho e resolução de problemas }\end{array}$ & 13.212 & $91,2(90,7-91,6)$ \\
\hline $\begin{array}{l}\text { EqSF realiza avaliação em conjunto com o apoiador institucional para a } \\
\text { qualificação do processo de trabalho }\end{array}$ & 6.830 & $47,1(46,3-47,9)$ \\
\hline $\begin{array}{l}\text { A equipe recebe apoio de outros profissionais para auxiliar ou apoiar na } \\
\text { resolução de casos considerados complexos }\end{array}$ & 13.609 & $93,9(93,5-94,3)$ \\
\hline \multicolumn{3}{|l|}{ Profissionais que realizam apoio matricial } \\
\hline NASF & 11.643 & $80,3(79,7-81,0)$ \\
\hline CAPS & 10.036 & $69,2(68,5-70,0)$ \\
\hline Vigilância em saúde & 12.050 & $83,2(82,5-83,8)$ \\
\hline Especialistas da rede & 11.414 & $78,8(78,1-79,4)$ \\
\hline Hospitais & 11.111 & $76,7(76,0-77,4)$ \\
\hline Polo de Academia da Saúde & 5.504 & $38,0(37,2-38,8)$ \\
\hline Centros especializados (reabilitação, idoso, obesidade e outros) & 8.041 & $55,5(54,7-56,3)$ \\
\hline \multicolumn{3}{|l|}{ Formas de acionar o apoio do NASF realizadas pelas EqSF } \\
\hline Encaminhamento por escrito & 9.880 & $68,2(67,4-68,9)$ \\
\hline Discussão de casos & 9.329 & $64,4(63,6-65,2)$ \\
\hline Consultas compartilhadas & 8.889 & $61,3(60,5-62,1)$ \\
\hline $\begin{array}{l}\text { Agendamento de consultas diretamente na agenda do profissional do } \\
\text { NASF }\end{array}$ & 9.237 & $63,7(63,0-64,5)$ \\
\hline Contato por e-mail e telefone & 8.874 & $61,2(60,4-62,0)$ \\
\hline O NASF atende as solicitações de apoio da sua equipe em tempo adequado & 7.052 & $48,7(47,8-49,5)$ \\
\hline $\begin{array}{l}\text { Existem critérios e formas definidas e pactuadas entre sua equipe e o NASF } \\
\text { para acionar apoio em situações imprevistas }\end{array}$ & 10.536 & $72,7(72,0-73,4)$ \\
\hline Em situações imprevistas, consegue o apoio do NASF & 11.355 & $78,4(77,7-79,0)$ \\
\hline
\end{tabular}


das EqSF realiza essas atividades, o que enfatiza a atribuição dos profissionais para discussão e planejamento das ações baseados nas realidades locais, conforme orientação da $\mathrm{PNAB}^{10}$.

Estudos destacam que a região Nordeste teve expansão dos serviços de APS por meio da ESF, com ênfase para as regiões menos favorecidas pela oferta assistencial pública e privada. A isso acrescenta-se também a melhoria na distribuição regional de serviços de média e alta complexidade. No contexto mais amplo de saúde, os determinantes sociais, o progresso democrático e o desenvolvimento sustentável estão intrinsecamente relacionados porque a mudança em um domínio afeta os outros. Dessa forma, ações integradas de saúde, sociais e econômicas são necessárias para o planejamento e apoio no processo de trabalho das equipes, visando a redução das desigualdades sociais e em saúde ${ }^{11,12}$.

O planejamento e o apoio da gestão são marcos importantes na organização dos serviços de saúde. No entanto, é preciso ter consciência e conhecimento das realidades e particularidades locais, respeitando os aspectos culturais, geográficos e sociais com foco na qualidade do serviço. Conhecendo esses aspectos, o planejamento irá conduzir o processo de reorientação das ações e serviços e, juntamente com o apoio institucional, desmistificará a avaliação externa como sendo de caráter punitivo, trazendo efeitos positivos para correção dos rumos e adequações das ações de saúde no processo de trabalho ${ }^{13}$.

Quanto ao monitoramento e análise dos indicadores e informações de saúde, destaca-se que a maior parte das equipes realiza esse acompanhamento. Estudo realizado em dois estados do Nordeste do país constatou que a maioria das equipes afirmou adotar essa atividade, com elevado percentual de utilização na organização do processo de trabalho na $\mathrm{AB}^{14}$.

Ademais, a quase totalidade das equipes afirmou realizar a autoavaliação para reorganização de seus processos de trabalho, detectando e reconhecendo suas potencialidades e fragilidades. Nesse sentido, em estudo realizado nos municípios do estado Espírito Santo, as equipes utilizam, em sua maioria, a autoavaliação para melhoria do acesso e qualidade da AB (AMAQ) como ferramenta de gestão para reorganização do seu trabalho. Outro estudo, realizado na $\mathrm{Pa}$ raíba, mostrou que o processo de autoavaliação é reconhecido pelos profissionais como oportunidade de identificar as fragilidades nos processos de trabalho das equipes ${ }^{15,16}$.

Os resultados desse trabalho permitem afirmar que a relação entre planejamento e organi- zação do cronograma de ações com o NASF é realizado de forma conjunta, destacando-se as fragilidades acerca da colaboração interprofissional entre profissionais das EqSF e do NASF, uma vez que ainda persistem visões de trabalho individualizadas e com pouca integração em todas as variáveis do contexto estudado ${ }^{17}$.

Estudos afirmam que o fortalecimento do entendimento do planejamento em equipe é essencial para a integralidade do cuidado e a realização de ações que efetivamente atendam às necessidades de saúde das pessoas e grupos, elevando o escopo de resolutividade do processo de trabalho ${ }^{18,19}$.

Evidencia-se que as equipes, em sua maioria, declararam receber apoio permanente da gestão para discutir o processo de trabalho. Já com relação à avaliação do trabalho das EqSF, em conjunto com o apoiador institucional, não foi considerável no estudo. Uma pesquisa que utilizou dados das regiões do Brasil atingiu melhores resultados quanto ao recebimento de apoio institucional e piores com relação à contribuição do apoiador no trabalho conjunto para a qualificação do processo de trabalho e enfrentamento dos problemas. A PNAB deixa claro que é competência municipal o apoio institucional às equipes na implantação, acompanhamento e qualificação da $\mathrm{AB}$, além da ampliação e concretização do processo de trabalho ${ }^{3,16}$.

No que se refere ao apoio matricial, a maioria das equipes recebe apoio de outros profissionais na resolução de casos complexos, principalmente da vigilância em saúde e do NASF sem periodicidade definida. Estudo que analisou a situação da $\mathrm{AB}$ por meio de municípios com distintos portes populacionais, salienta que, em municípios de pequeno porte populacional, a oferta de serviços na $\mathrm{AB}$ mostra-se restrita e pouco resolutiva quando comparada às EqSF inseridas em contextos municipais mais favoráveis que recebem apoio de outros profissionais ${ }^{20}$.

No entanto, o presente estudo mostrou que $93,9 \%$ das equipes referem receber apoio para a resolução de casos considerados complexos na organização do processo de trabalho. Esses dados merecem atenção, uma vez que a organização do processo de trabalho, através do apoio matricial, deve ser pensada a fim de expandir o escopo de atuação das EqSF, trazendo uma equipe interdisciplinar e aumentando o potencial de integralidade e de resolutividade dos atendimentos, seja como referência ou incorporando-os nas equipes, estabelecendo uma relação horizontal e dialógica entre profissionais. Para tal fim, é necessário construir várias linhas de transversalidade e 
ordenar essa relação entre equipe de referência, apoios e especialistas com base em procedimentos dialógicos ${ }^{21}$.

Os dados mostraram que o NASF tem uma forte atuação no planejamento e desenvolvimento de ações de forma integrada com EqSF, assim como organiza a sua agenda de forma definida e pactuada para situações imprevistas dando o apoio nessas eventualidades ${ }^{17}$.

Vale salientar que, apesar da análise do presente estudo ter sido realizada na região Nordeste, suas inferências podem identificar em alguns contextos quais as necessidades e o que se encontra com maior fragilidade de uma atenção especificada referente ao processo de trabalho das equipes de $\mathrm{AB}$, o que pode ser aplicado em outras partes do país.

$\mathrm{O}$ estudo reconhece que, mediante os indicadores avaliados do PMAQ-AB, ao nosso conhecimento, este é o primeiro estudo realizado com os dados do $3^{\circ}$ ciclo a incluir dados sobre o processo de trabalho das equipes de $\mathrm{AB}$ com embasamento nas ações de planejamento da equipe e apoio institucional, acesso das equipes ao NASF, articulação entre ESF e NASF e apoio matricial para as equipes de $\mathrm{AB}$ na região Nordeste.

\section{Colaboradores}

GT Silva, FO Carvalho, APGF Vieira-Meyer, GMS Gomes, LMMR Bezerra, RV Camelo, VC Barbosa Filho participaram igualmente da concepção, delineamento, análise e interpretação dos dados, redação do artigo ou a sua revisão crítica, e aprovação da versão a ser publicada.

\section{Conclusão}

Por fim, dada a carência de estudos que se utilizam de abordagens quantitativas para avaliar as ações do processo de trabalho no contexto da ESF na região Nordeste, os resultados aqui apresentados podem ser de grande utilidade para gestores, profissionais e acadêmicos, que de alguma forma estejam relacionados à atenção primária à saúde. As EqSF têm planejado e organizado o seu processo de trabalho com a realização de autoavaliação, monitoramento e análise de indicadores de informações da saúde, apresentando ainda bons resultados nas ações de apoio da gestão e apoio matricial.

O estudo conferiu uma boa representatividade de seus resultados, algumas potencialidades e fragilidades do processo de trabalho das EqSF que atuam na região Nordeste, exigindo esforços para a mudança da realidade ainda fragmentada e verticalizada, favorecendo um panorama de trabalho que sinaliza para melhores resultados no processo avaliativo do PMAQ-AB, uma vez que $A B$ não existe sem processo de trabalho em equipe e, portanto, sem um sujeito coletivo de corresponsabilidade, tanto na deliberação quanto no planejamento para a organização de serviços de saúde adequados para a população. 


\section{Referências}

1. World Health Organization (WHO). United Nations Children's Fund (UNICEF). Declaration of Astana [Internet]. 2018 [acessado 2019 Ago 23]. Disponível em: https://www.who.int/docs/default-source/primaryhealth/declaration/gcphc-declaration.pdf

2. Facchini LO, Tomasi E, Dilélio AS. Qualidade da Atenção Primária à Saúde no Brasil: avanços, desafios e perspectivas. Saúde Debate 2018; 42(spe):208-223.

3. Brasil. Ministério da Saúde (MS). Política Nacional de Atenção Básica (PNAB). Brasília: MS 2012.

4. Sarti TD, Campos CEA, Zandonade E, Ruschi GEC, Maciel ELN. Avaliação das ações de planejamento em saúde empreendidas por equipes de saúde da família. Cad Saúde Pública 2012; 3(28):537-548.

5. Ferreira IRC, Moysés SJ, França BHS, Carvalho ML Moysés ST. Percepções de gestores locais sobre a intersetorialidade no Programa Saúde na Escola. Rev Bras Educ 2014; 19(56):61-76.

6. Lima CA, Moreira KS, Costa GS, Maia RS, Pinto MQC, Vieira MA, Costa SM. Avaliação do processo de trabalho entre equipes de saúde da família de um município de Minas Gerais, Brasil. Trab Educ Saúde 2019; 17(1):1-20.

7. Brasil. Ministério da Saúde (MS). Portaria no 1.654 , de 19 de julho de 2011. Institui, no âmbito do Sistema Único de Saúde, o Programa Nacional de Melhoria do Acesso e da Qualidade da Atenção Básica (PMAQ-AB) e o Incentivo Financeiro do PMAQ-AB, denominado Componente de Qualidade do Piso de Atenção Básica Variável - PAB Variável. Diário Oficial da União; 2011.

8. Brasil. Ministério da Saúde (MS). Secretaria de Atenção à Saúde. Departamento de Atenção Básica. Saúde Mais Perto de Você. Programa Nacional da Melhoria do Acesso e da Qualidade da Atenção Básica: manual instrutivo. Brasília: MS; 2012.

9. Instituto Brasileiro de Geografia e Estatística (IBGE). Censo demográfico 2010. Rio de Janeiro: IBGE; 2019.

10. Medrado JRS, Casanova AO, Oliveira CCM. Estudo avaliativo do processo de trabalho das Equipes de Atenção Básica a partir do PMAQ-AB. Saúde Debate 2015; 39(107):1033-1043.

11. Albuquerque MV, Viana ALD, Lima LD, Ferreira MP, Fusaro ER, Iozzi FL. Desigualdades regionais na saúde: mudanças observadas no Brasil de 2000 a 2016. Cien Saude Colet 2017; 22(4):1055-1064.

12. Fiorati RC, Souza LB, Cândido FCA, Silva LNF, Finzeto LC, Alves LS, Berra TZ, Arcêncio RA. Iniquidades sociais e intersetorialidade: desafio à atenção primária à saúde. Rev Enferm UFPE 2018; 12(6):1705-1716.

13. Rodrigues V, Santos CRI, Pereira MU. A experiência de planejar e operacionalizar o PMAQ-AB no estado do Acre. Saúde Debate 2014; 38(spe):173-181.
14. Carvalho MF, Vasconcelos MIO, Silva ARV, Vieira APGF. Utilização de monitoramento e análise de indicadores na Atenção Primária à Saúde. Sanare 2017; 16(1):67-73.

15. Sampaio J, Moraes MN, Marcolino EC, Castro ID, Gomes LB, Clementino FS. PMAQ-AB: a experiência local para a qualificação do programa nacional. Rev Enferm UFPE 2016; 10(5):4318-4328.

16. Cruz MM, Souza RBC, Torres RMC, Abreu DMF, Reis AC, Gonçalves AL. Usos do planejamento e autoavaliação nos processos de trabalho das equipes de Saúde da Família na Atenção Básica. Saúde Debate 2014; 38(spe):124-139.

17. Lima RSA, Nascimento JA, Ribeiro KSQS, Sampaio J. $\mathrm{O}$ apoio matricial no trabalho das equipes dos Núcleos de Apoio à Saúde da Família: análise a partir dos indicadores do $2^{\circ}$ ciclo do Programa Nacional de Melhoria do Acesso e da Qualidade. Cad Saúde Colet 2019; 27(1):25-31.

18. Silva ICB, Silva LAB, Lima RSA, Rodrigues JA, Valença AMG, Sampaio J. Processo de trabalho entre a Equipe de Atenção Básica e o Núcleo de Apoio à Saúde da Família. Rev Bras Med Fam Comunidade 2017; 12(39):1-10.

19. Matuda CG, Pinto NRS, Martins CL, Frazão P. Colaboração interprofissional na estratégia saúde da família: implicações para a produção do cuidado e a gestão do trabalho. Cien Saude Colet 2015; 20(8):2511-2521.

20. Rocha PAS, Leite FMC, Barbosa, LFA, Lima RCD Programa Nacional de Melhoria da Qualidade e do Acesso da Atenção Básica: avaliando o processo de trabalho das equipes do Espírito Santo. APS Rev 2019; 1(2):141-151.

21. Santos AF, Machado ATGM, Reis CMR, Abreu DMX, Araújo LHL, Rodrigues SC, Lima AMLD, Jorge AO, Sobrinho DF. Apoio institucional e matricial e sua relação com a atenção básica. Rev Saude Publica 2015; $49(54): 1-7$

Artigo apresentado em 07/08/2020

Aprovado em 22/02/2021

Versão final apresentada em 24/02/2021

Editores-chefes: Romeu Gomes, Antônio Augusto Moura da Silva 
